\title{
RESÍDUOS DE BENOMIL EM MAMÃO (Carica papaya L.)TRATADO EM PÓS-COLHEITA
}

\author{
JORGE JOSÉ DO VALE OLIVEIRA *
}

\begin{abstract}
Para avaliar os níveis de resíduos de Benomil em mamão (Carica papaya L.), tratado em pós-colheita, utilizou-se Benlate 500 PM (250 mg/L, $500 \mathrm{mg} / \mathrm{L}$ e $1000 \mathrm{mg} / \mathrm{L})$ por imersão em diferentes combinações, com e sem o emprego de cera, totalizando oito tratamentos. Em seguida os mamões foram armazenados a $12{ }^{\circ} \mathrm{C}$ e $85-90 \%$ de unidade relativa do ar (UR) durante 21 dias. Os níveis de Benomil foram analisados por cromatografia a líquido de alta eficiência com detector de ultravioleta (UV) a $286 \mathrm{~nm}$, na polpa e na casca após $0,1,4,7$ e 21 dias de armazenamento. Não foram detectados resíduos de Benomil na polpa de acordo com o limite de quantificação do método $(0,3 \mathrm{mg} /$ $\mathrm{kg}$ ), porém, níveis significativos foram detectados na casca, com degradação na faixa de $55 \%-84 \%$ entre 0 e 21 dias de armazenamento.
\end{abstract}

PALAVRAS-CHAVE: MAMÃO; BENOMIL; AGROTÓXICOS-RESÍDUOS.

\section{INTRODUÇÃO}

O Brasil é o maior produtor mundial de mamão papaya, com produção da ordem de 1.500 .000 toneladas ao ano, sendo mais de $99 \%$ absorvida pelo mercado interno. Embora a exportação de mamão papaya seja modesta em relação ao consumo interno, o mercado externo é atraente e promissor a curto e médio prazos. Desta forma, o mamão papaya encontra-se listado na pauta de exportação brasileira, com tendência de crescimento futuro da ordem de milhões de dólares (SILVA, 1998).

Tratando-se de fruta tropical, o mamão requer cuidados especiais. É altamente perecível em temperatura ambiente e muito susceptível à doenças e podridões causadas por fungos. Em razão de fatores climáticos e econômicos muitas vezes não é possível desenvolver controle fitossanitário adequado no campo. O tratamento pós-colheita em frutas é

* Pesquisador Científico, Instituto de Tecnologia de Alimentos, Campinas, SP.

(e-mail: jorgejvo@ital.org.br). 
usado para aumentar seu tempo de vida e preservar sua qualidade durante a estocagem, o transporte e a comercialização, principalmente, quando as frutas são destinadas para exportação (PAPADOPOULOUMOURKIDOU, 1991).

Para combater as doenças, que inviabilizam a comercialização e o consumo do mamão empregam-se fungicidas no tratamento fitossanitário, tanto em pré-colheita como em pós-colheita. Assim como os fungicidas podem apresentar eficácia profilática, dependendo da forma de aplicação, sua utilização não criteriosa pode acarretar males à saúde do consumidor quando ingeridos em grande quantidade (SPADOTTO et al., 1998; NUNES e RIBEIRO, 1999; OLIVEIRA e TOLEDO, 2000).

O objetivo deste trabalho foi avaliar os níveis de residuais de Benomil na casca e na polpa de mamões papaya tratados em pós-colheita por imersão.

\section{MATERIAL E MÉTODOS}

\subsection{TRATAMENTOS}

Os mamões (Carica papaya L.) colhidos no campo, transportados em engradados de madeira até o laboratório, foram selecionados previamente quanto a integridade, firmeza e sanidade dos frutos. Posteriormente foram submetidos a oito tratamentos envolvendo banho frio e térmico, imersão em Benomil, com ou sem aplicação de cera com compressor (Tabela 1).

\section{TABELA 1 - DESCRIÇÃO DOS TRATAMENTOS}

\begin{tabular}{|c|c|c|c|}
\hline Tratamentos & $\begin{array}{l}\text { gua (imers }{ }^{\mathrm{o}} \text { ) } \\
47-49 \text { "C/5min }\end{array}$ & $\begin{array}{c}\left.\text { gua (imers }{ }^{\mathrm{a}} \mathrm{o}\right) \\
\text { Temperatura Ambiente } \\
5 \mathrm{~min}\end{array}$ & $\begin{array}{l}\text { Aplica a o de cera } \\
\text { Stafresh }(1: 2)\end{array}$ \\
\hline T1 & $(+)$ & $(+)$ & $(+)$ \\
\hline T2 & $(-)$ & $(+)$ & $(-)$ \\
\hline T3 & $+/ F(1000)$ & $(+)$ & $(+)$ \\
\hline $\mathrm{T} 4$ & $(+)$ & $(+) / F(1000)$ & $(+)$ \\
\hline T5 & $(+)$ & $(+)$ & $(+) / F(1000)$ \\
\hline T6 & $(+) / F(500)$ & $(+)$ & $(+) / F(500)$ \\
\hline T7 & $(+)$ & $(+) / F(500)$ & $(+) / F(500)$ \\
\hline T8 & $(+) / F(500)$ & $(+) / F(250)$ & $(+) / F(250)$ \\
\hline
\end{tabular}

$(+)$ = submetido ao tratamento indicado; $(-)$ = não submetido ao tratamento indicado; $\mathrm{F}(250), \mathrm{F}(500), \mathrm{F}(1000)=$ Benomil a $250 \mathrm{mg} / \mathrm{L}, 500 \mathrm{mg} / \mathrm{L}, 1000 \mathrm{mg} / \mathrm{L}$, respectivamente . T1 e T2 = Controle. 


\subsection{ARMAZENAMENTO}

Quarenta frutos submetidos aos tratamentos foram embalados em cinco caixas de papelão, totalizando oito frutos por caixa. Em seguida as caixas foram armazenadas durante $0,1,4,14$ e 21 dias em câmara fria a $12{ }^{\circ} \mathrm{C}$ e $85-90 \%$ de umidade relativa do ar (UR). Após estes dias de armazenamento, cada caixa com os oito mamões foi reservada para as análises de resíduos de Benomil.

\subsection{PADRÃO}

O padrão de Benomil foi obtido da DuPont com índice de pureza de $99,2 \%$. Foram preparados padrões de Carbendazin, quantidade equivalente a $100 \mathrm{mg} / \mathrm{L}$ de Benomil. O Benomil foi dissolvido em acetona e deixado em temperatura ambiente, durante 8 horas para total conversão a Carbendazin. Para elaboração da curva padrão, preparou-se, por diluição com metanol, padrões de Carbendazin equivalentes a $0,3 \mathrm{mg} / \mathrm{L}, 0,5 \mathrm{mg} /$ $\mathrm{L}, 1,0 \mathrm{mg} / \mathrm{L}, 1,5 \mathrm{mg} / \mathrm{L}, 2,0 \mathrm{mg} / \mathrm{L}$ e $3,0 \mathrm{mg} / \mathrm{L}$ de Benomil.

\subsection{MÉTODO DE EXTRAÇÃO DE RESÍDUOS DE BENOMIL}

Após o armazenamento a casca e a polpa dos mamões foram separadas, homogeneizadas em processador, acondicionadas em frascos de vidro, tampados e armazenadas em freezer $\left(-20^{\circ} \mathrm{C}\right)$ até a ocasião das análises. Os resíduos de Carbendazin foram quantificados nas amostras pelo método modificado de BARDALAYE e WHELLER, 1985, e as concentrações multiplicadas por 1,52 para conversão em concentrações de Benomil (WHITE e KILGORE, 1972; CHIBA, 1977). Utilizando-se balança semi-analítica foram pesados em erlenmeyer $5 \mathrm{~g}$ de amostra $\mathrm{e}$ adicionaram-se $50 \mathrm{~mL}$ de metanol:tampão fosfato $(2,4441 \mathrm{~g}$ de $\mathrm{Na} 2 \mathrm{HPO} 4.7 \mathrm{H} 2 \mathrm{O}$ e 0,3351 g de K2HPO4 em 1 litro de água filtrada em filtro millipore) (60:40). Agitou-se a mistura durante 30 minutos e filtrou-se o extrato por gravidade em papel de filtro Whatman $n-5$, utilizando funil de vidro como suporte. O erlenmeyer e o filtro foram lavados com aproximadamente $50 \mathrm{~mL}$ da mistura metanol:tampão (60:40). O filtrado foi transferido para funil de separação de $500 \mathrm{~mL}$, sendo adicionados $50 \mathrm{~mL}$ de clorofórmio. Agitou-se o funil vigorosamente durante 2 minutos para separação da fase orgânica. A fase aquosa foi novamente particionada com $50 \mathrm{~mL}$ de clorofórmio, sendo as duas fases de clorofórmio recolhidas e lavadas com $30 \mathrm{~mL}$ de água. As duas fases aquosas foram descartadas. 
O clorofórmio foi evaporado em rotavapor e ressuspendido com metanol a $1 \mathrm{~mL}$ e $5 \mathrm{~mL}$, de acordo com a concentração de Benomil em cada amostra, e em seguida filtrados com filtros millipore de $0,45 \mu \mathrm{m}$ e injetados em cromatógrafo a líquido.

\subsection{CONDIÇÕES CROMATOGRÁFICAS}

Utilizou-se cromatógrafo a líquido de alta eficiência Varian, modelo 9002, detector ultravioleta-visível a $286 \mathrm{~nm}$ com sensibilidade de RCDR/ AUFS 0,01, modelo 9050, acoplados a workstation modelo Star. Empregouse coluna de aço inoxidável de $12,5 \mathrm{~cm}$ de comprimento por $4,6 \mathrm{~mm}$ de diâmetro interno empacotada com RP-18 $(5 \mu \mathrm{m})$ e válvula reodyne com loop de $20 \mu \mathrm{L}$. Empregou-se como fase móvel a mistura metanol:tampão fosfato (60:40) com fluxo programável de $0,75 \mathrm{~mL} / \mathrm{min}$ ( 0 a 8 minutos), de $0,75 \mathrm{~mL} / \mathrm{min}$ a $1,0 \mathrm{~mL} / \mathrm{min}$ (8 a 10 minutos) e de $1,0 \mathrm{~mL} / \mathrm{min}$ a $0,75 \mathrm{~mL} /$ min (10 a 15 minutos), com tempo de corrida total de 15 minutos em temperatura ambiente.

\section{RESULTADOS E DISCUSSÃO}

Os resultados das fortificações e recuperações de Benomil na casca e polpa dos mamões encontram-se na Tabela 2. Os cromatogramas dos extratos da casca e da polpa dos mamões isentos da aplicação de Benomil não apresentaram pico interferente no tempo de retenção deste fungicida.

\section{TABELA 2 - RESULTADOS DAS RECUPERAÇÕES DE BENOMIL EM MAMÃO}

\begin{tabular}{c|c|c|c|c}
\hline MAM^ & Massa $(\mathrm{g})$ & $\begin{array}{c}\text { Benomil } \\
\text { Adicionado }(\mathrm{g})\end{array}$ & $\begin{array}{c}\text { Fortifica a o } \\
(\mathrm{g} / \mathrm{g})\end{array}$ & Recupera o (\%) \\
\hline \multirow{3}{*}{ Casca } & 5 & 5 & 1,0 & 102 \\
& 5 & 5 & 1,0 & 109 \\
& 5 & 2,5 & 0,5 & 87 \\
& 5 & 2,5 & 0,5 & 93 \\
& 5 & 1,5 & 0,3 & 71 \\
& 5 & 5 & 1,0 & 71 \\
& 5 & 5 & 1,0 & 120 \\
& 5 & 5 & 1,0 & 82 \\
& 5 & 2,5 & 0,5 & 100 \\
& 5 & 2,5 & 0,5 & 114 \\
& 5 & 2,5 & 0,5 & 88 \\
\hline
\end{tabular}

As amostras de casca e polpa foram fortificadas com quantidades de Carbendazin equivalentes as concentrações de $1,0 \mathrm{mg} / \mathrm{kg}, 0,5 \mathrm{mg} / \mathrm{kg}$ e $0,3 \mathrm{mg} / \mathrm{kg}$ de Benomil. 
Os níveis de resíduos de Benomil na casca do mamão encontramse na Tabela 3. Não foram detectados resíduos de Benomil nas polpas dos mamões de acordo com o limite de quantificação do método $(0,3 \mathrm{mg} /$ $\mathrm{kg}$ ) empregado. Este situa-se muito abaixo dos Limites Máximos de Resíduos (LMR) de pesticidas estabelecidos pela legislação brasileira e pelo CODEX para outras frutas (Tabela 4), considerando que não há registro deste fungicida para mamão (BRASIL, 2000; FAO/WHO, 2000).

\section{TABELA 3 - NÍVEIS DE RESÍDUOS DE BENOMIL (mg/kg) NAS CASCAS DOS MAMÕES}

\begin{tabular}{c|c|c|c|c|c|c|c|c}
\hline A(dias) & T1 & T2 & T3 & T4 & T5 & T6 & T7 & T8 \\
\hline 0 & Nd & Nd & 27,43 & 13,26 & 10,56 & 23,30 & 13,29 & 27,88 \\
1 & Nd & Nd & - & 10,89 & 10,02 & 23,11 & - & 17,02 \\
4 & Nd & Nd & 29,01 & 11,38 & 6,11 & 17,68 & 13,49 & 13,13 \\
14 & Nd & Nd & 16,46 & 6,24 & - & 7,50 & 6,32 & 7,55 \\
21 & Nd & Nd & 14,40 & 2,15 & 4,66 & 7,22 & 4,36 & 7,24 \\
\hline
\end{tabular}

$\mathrm{Nd}=$ não detectado (abaixo do limite de quantificação do método). $\mathrm{T}$ = Tratamento . $\mathrm{A}=$ Armazenamento.

A não detecção de resíduos de Benomil na polpa do mamão está de acordo com os resultados obtidos em outras pesquisas realizadas com polpa de manga e abacaxi (SCALON et al., 1996; OLIVEIRA et al., 1997), podendo-se afirmar que as cascas desempenharam função de escudo protetor à penetração dos resíduos de Benomil. Trabalhos realizados por outros pesquisadores também confirmam que grande parte de Benomil permaneçe na casca das frutas (CANO et al., 1987; SCALON et al., 1996; OLIVEIRA et al., 1997).

\section{TABELA 4 - LIMITE MÁXIMO DE RESÍDUOS (LMR) DE PESTICIDAS PARA O BENOMIL EM DIVERSAS FRUTAS}

\begin{tabular}{cccc}
\hline Cultura & \multicolumn{2}{c}{ LMR $(\mathrm{mg} / \mathrm{kg})$} & CarCficia (dias) \\
\hline Abacaxi & Legisla ${ }^{\circ}$ o Brasileira & CODEX & 1 \\
Banana & 2,0 & $\mathrm{Nr}$ & 3 \\
Citros & 1,0 & 1,0 & 1 \\
Ma a & 5,0 & $\mathrm{Nr}$ & 1 \\
Manga & 5,0 & $\mathrm{Nr}$ & 1 \\
Mela 0 & 2,0 & 2,0 & 3 \\
POssego & 0,5 & 2,0 & 1 \\
Uva & 5,0 & $\mathrm{Nr}$ & 18 \\
\hline
\end{tabular}

${ }^{*}$ Como Carbendazin. $\mathrm{Nr}=$ não registrado.

FONTE: BRASIL, 2000 e FAO/WHO, 2000. 
Não foram detectados resíduos de Benomil nos tratamentos $1 \mathrm{e}$ 2 da casca, uma vez que estas amostras foram utilizadas como testemunhas para controle analítico do perfil cromatográfico. Ocorreu degradação nos níveis de resíduos de Benomil, em todos os tratamentos, entre os tempos de armazenamento de 0 e 21 dias, na faixa de $47 \%$ a $83 \%$. Nas mesmas condições de temperatura e umidade relativa do ar (UR), OLIVEIRA et al. (1997) também constataram degradação de Benomil da ordem de $22 \%$ na casca de mangas após 29 dias de armazenamento.

Os valores encontrados para os tratamentos 3, 5 e 7 (nos dias de armazenamento de 1,14 e 1 dia, respectivamente) não foram compatíveis com as faixas residuais de cada tratamento. É possível que o manuseio das amostras durante a homogeneização possa ter influenciado a discrepância entre os valores, motivo pelo qual não foram apresentados.

\title{
4 CONCLUSÃO
}

Embora a legislação brasileira não estabeleça limites máximos de resíduos para o Benomil em mamão, as polpas analisadas estariam próprias para o consumo de acordo com o limite de detecção do método empregado (situado muito abaixo dos limites desse fungicida fixados para outras frutas).

A aplicação de altas concentrações de Benomil em pós-colheita revelou níveis significativos de resíduos na casca dos mamões, com degradação na faixa de $55 \%$ a $84 \%$ entre 0 e 21 dias de armazenamento.

\begin{abstract}
BENOMYL RESIDUES IN PAPAYA (Carica papaya L.) TREATED IN POSTHARVEST

In order to evaluate the residues levels of Benomyl in papaya (Carica papaya L.), treated in post-harvest, Benlate $500 \mathrm{PM}(250 \mathrm{mg} / \mathrm{L} ; 500 \mathrm{mg} / \mathrm{L} \mathrm{e} 1000 \mathrm{mg} / \mathrm{L})$ was applied by dipping in different combinations with and without wax, totalyzing eight treatments. After this the papayas were stored at $12^{\circ} \mathrm{C}$ and $85-90 \%$ relative humidity during 21 days. The benomyl levels were analyzed by high performance liquid chromatography at $286 \mathrm{~nm}$, in the pulp and in the peel after $0,1,4,14$ and 21 days of storage. No Benomyl residues were detected in the pulp within the quantification limit of the applied method $(0,3 \mathrm{mg} / \mathrm{kg})$ but significative levels were detected in the peel, with degradation in the range of $55 \%-84 \%$ between 0 and 21 days of storage.

KEY-WORDS: PAPAYA; BENOMYL; PESTICIDES-RESIDUES.
\end{abstract}

\section{REFERÊNCIAS}

1 BARDALAYE, P. C.; WHELLER, W. B. Simplified method for the 
clean-up and reversed-phase high perfomance liquid chromatografic determination of benomyl in mangoes. Journal of Chromatography, v. 330, n. 2, p. 403-407, 1985.

2 BRASIL. Ministério da Saúde. Monografias de uso agropecuário e domissanitários: portarias e resoluções do Ministério da Saúde. 2. ed. São Paulo: ILSI, 2000.

3 CANO, P.; DE LA PLAZA, J. L.; MUNHOZ-DELGADO, L. Determination and persistence of several fungicides in post-harvest treated apples during their cold storage. Journal of Agricultural and Food Chemistry, v. 35, n. 1, p. 144-147, 1987.

4 CHIBA, M. A rapid spectrophotometric method for the simultaneous determination of intact benomyl and its degradation product, methyl 2-benzimidazolecarbamate (MBC), in organic solvents and water. Journal of Agricultural and Food Chemistry, v. 25, n. 2, p. 368373, 1977.

5 FAO/WHO. Codex alimentarius. $2^{\text {nd }}$ ed. Rome, 2000. v. $2 \mathrm{~B}, \mathrm{p}$. 101-104.

6 NUNES, G. S.; RIBEIRO, M. C. Pesticidas: uso, legislação e controle. Pesticidas: Revista de Ecotoxicologia e Meio Ambiente, Curitiba, v. 9, p. 31-34, 1999.

7 OLIVEIRA, J. J. V.; SIGRIST, J. M. M.; SPULVERATO, C. A.; POHLMANN, M. Degradação de Benomil em mangas Palmertratadas em pós-colheita. Pesticidas: Revista de Ecotoxicologia e Meio Ambiente, Curitiba, v. 7, p. 25-32, 1997.

8 OLIVEIRA, J. J. V.; TOLEDO, M. C. F. Níveis residuais de thiabendazole e imazalil em frutas de laranja-pêra tratadas por imersão em pós-colheita. Revista Brasileira de Fruticultura, v. 22, n. 3, p. 339-344, 2000.

9 PAPADOPOULOU-MOURKIDOU, E. Postharvest applied agrochemicals and their residues in fresh fruits and vegetables. Journal of the Association of Official Analytical Chemists, v. 74, n. 5, p. 745-765, 1991.

10 SILVA, C.E.L. EUA vão importar mamão papaia do ES. Folha de São Paulo, 20/01/98. p. 4. (Seção Dinheiro). 
11 SCALON, S. P. Q.; OLIVEIRA, J. J. V.; LIMA, L. C. O. Resíduos de Benomil em polpas de mangas haden armazenadas em atmosfera modificada. Pesticidas: Revista Técnico Científica, Curitiba, v. 6, p. 43-54, 1996.

12 SPADOTTO, C. A.; GOMES, M. A. F.; RODRIGUES, G. S. Uso de agrotóxicos nas diferentes regiões brasileiras: subsídio para a geomedicina. Pesticidas: Revista de Ecotoxicologia e Meio Ambiente, Curitiba, v. 8, p. 111-126, 1998.

13 WHITE, E. R.; KILGORE, W. W. Determination of systemic MBC residues in food crops treated with benomyl fungicide. Journal of Agricultural and Food Chemistry, v. 20, n. 6, p. 1230-1232, 1972. 